Supporting Information

\title{
Shapeable Aerogels of Metal-Organic-Frameworks Supported by Aramid Nanofibrils for Efficient Adsorption and Interception
}

\author{
Lu Zong ${ }^{\dagger,}$, Yongqiang Yang ${ }^{\dagger,}$, Hui Yang ${ }^{1}$, Xiaochen $W u^{1,3,{ }^{*}}$
}

${ }^{1}$ Department of Pharmacy, College of Chemical Engineering, Qingdao University of Science and Technology, Qingdao 266042, China

${ }^{2}$ National Graphene Products Quality Supervision and Inspection Center (Jiangsu), Jiangsu Province Special Equipment Safety Supervision Inspection Institute-Branch of Wuxi, Yanxin Road 330, Wuxi 214174, PR China

${ }^{3}$ Key Laboratory of Pharmaceutical Research for Metabolic Diseases, Qingdao University of Science and Technology, Qingdao 266042, China

Corresponding Author: (X. Wu)wxcguest@126.com

†L. Zong and Y. Yang contributed equally to this work. 


\section{Additional Experimental}

ZIF-67, HKUST-1 and Ni-HITP hybrid aerogels were obtained in a similar way as ZIF-8 hybrid aerogels. For example, ZIF-67 hybrid aerogels were acquired with 4.9 $\mathrm{mg} / \mathrm{mL}$ of cobalt nitrate and $5.5 \mathrm{mg} / \mathrm{mL}$ of 2 -methylimidazole at $80{ }^{\circ} \mathrm{C}$ for $12 \mathrm{~h}$ in methanol, HKUST-1 decorated aerogels were acquired with $4.1 \mathrm{mg} / \mathrm{mL}$ of copper nitrate and $4.1 \mathrm{mg} / \mathrm{mL}$ of trimesic acid at $25^{\circ} \mathrm{C}$ for $12 \mathrm{~h}$ in ethanol, while Ni-HITP hybrid aerogels were acquired with $2.2 \mathrm{mg} / \mathrm{mL}$ of nickel nitrate, $3.3 \mathrm{mg} / \mathrm{mL}$ of HITP and $1.3 \mathrm{M}$ of $\mathrm{NH}_{3} \cdot \mathrm{H}_{2} \mathrm{O}$ in $\mathrm{H}_{2} \mathrm{O}$ at $70{ }^{\circ} \mathrm{C}$ for $12 \mathrm{~h}$ (ref: ACS Nano 2019, 13 (8), 95789586). 

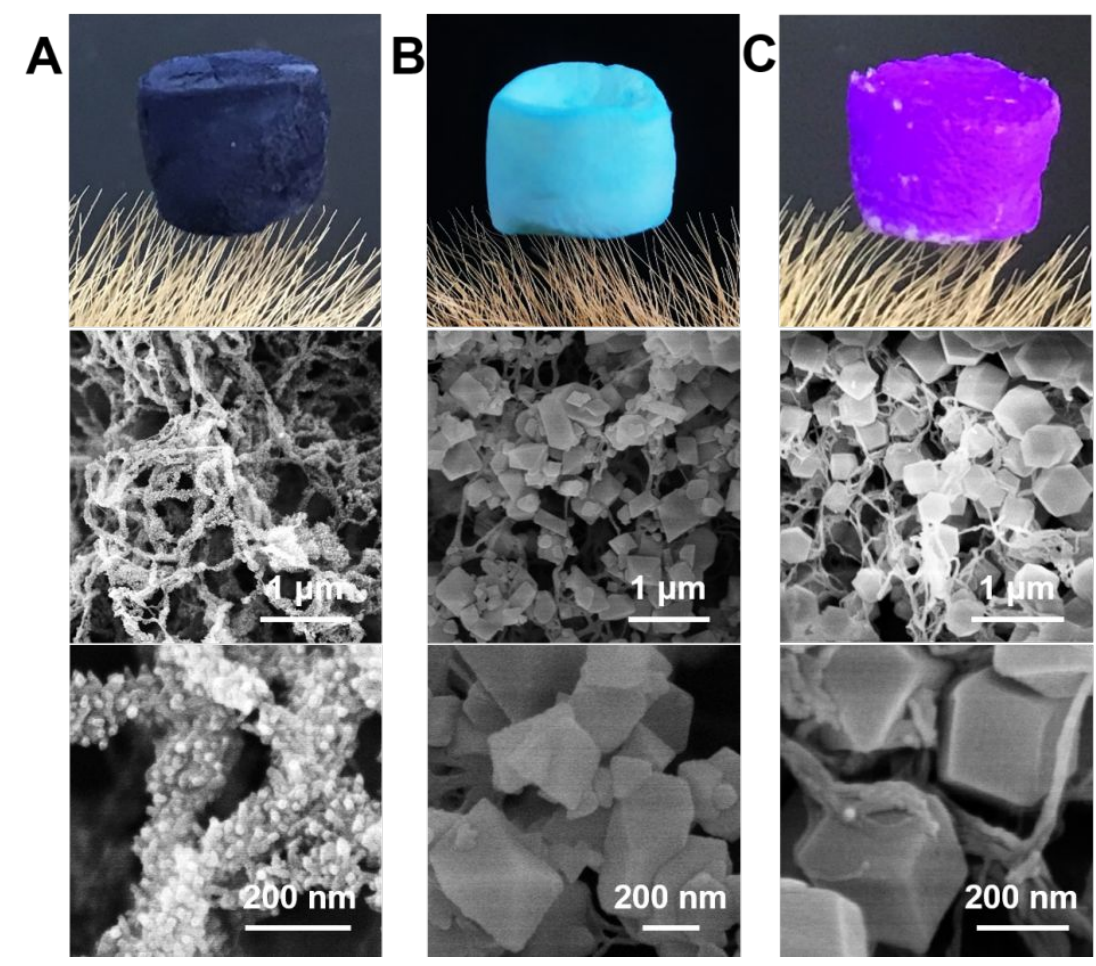

Figure S1. Photographs and SEM images of the MOFs hybrid aerogels. (A) Ni-HITP, (B) HKUST-1, and (C) ZIF-67 hybrid aerogels.

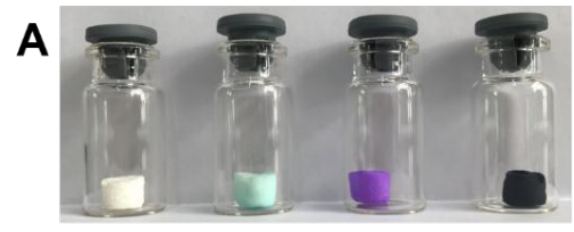

B
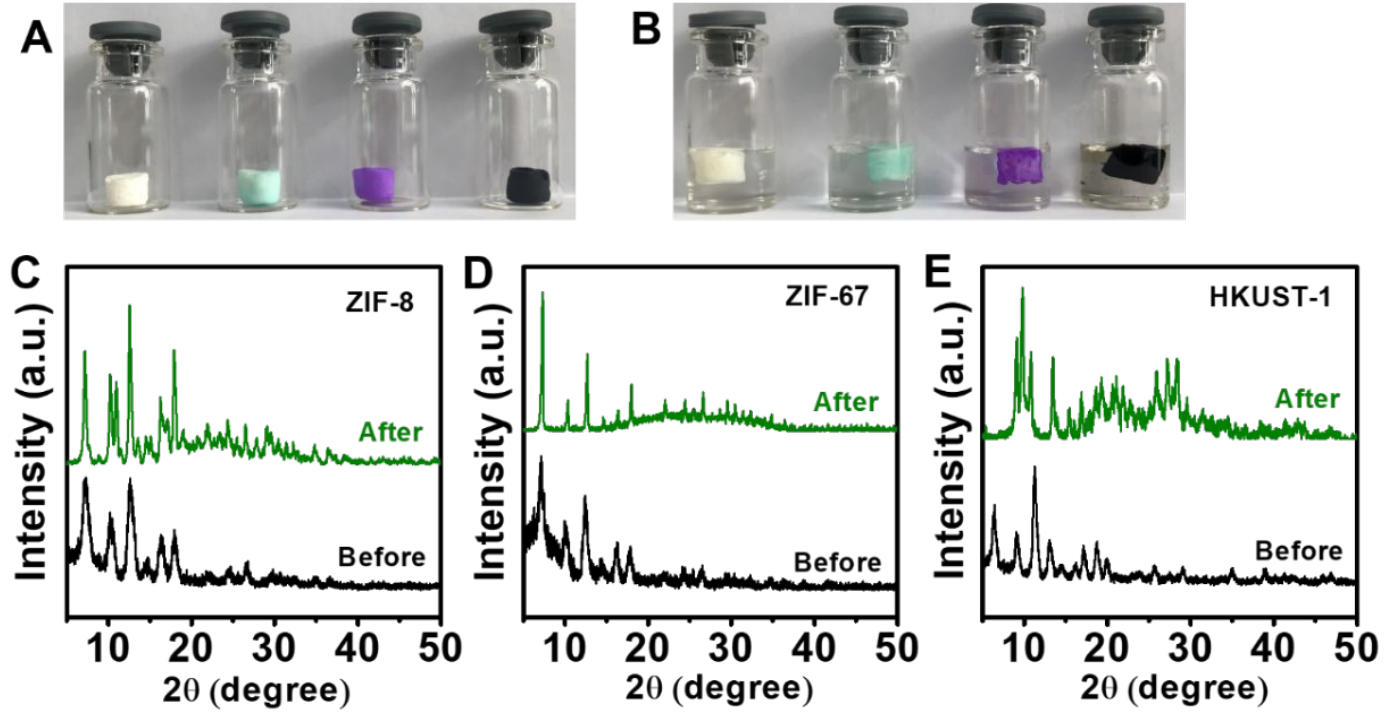

Figure S2. (A-B) Photographs of MOFs hybrid aerogels under (A) dry condition and immersing in $\mathrm{H}_{2} \mathrm{O}$ for $24 \mathrm{~h}$. From left to right: ZIF-8, HKUST-1, ZIF-67 and Ni-HITP hybrid aerogels. (C-E) XRD patterns of MOFs hybrid aerogels before and after immersing in $\mathrm{H}_{2} \mathrm{O}$ for $24 \mathrm{~h}$. 

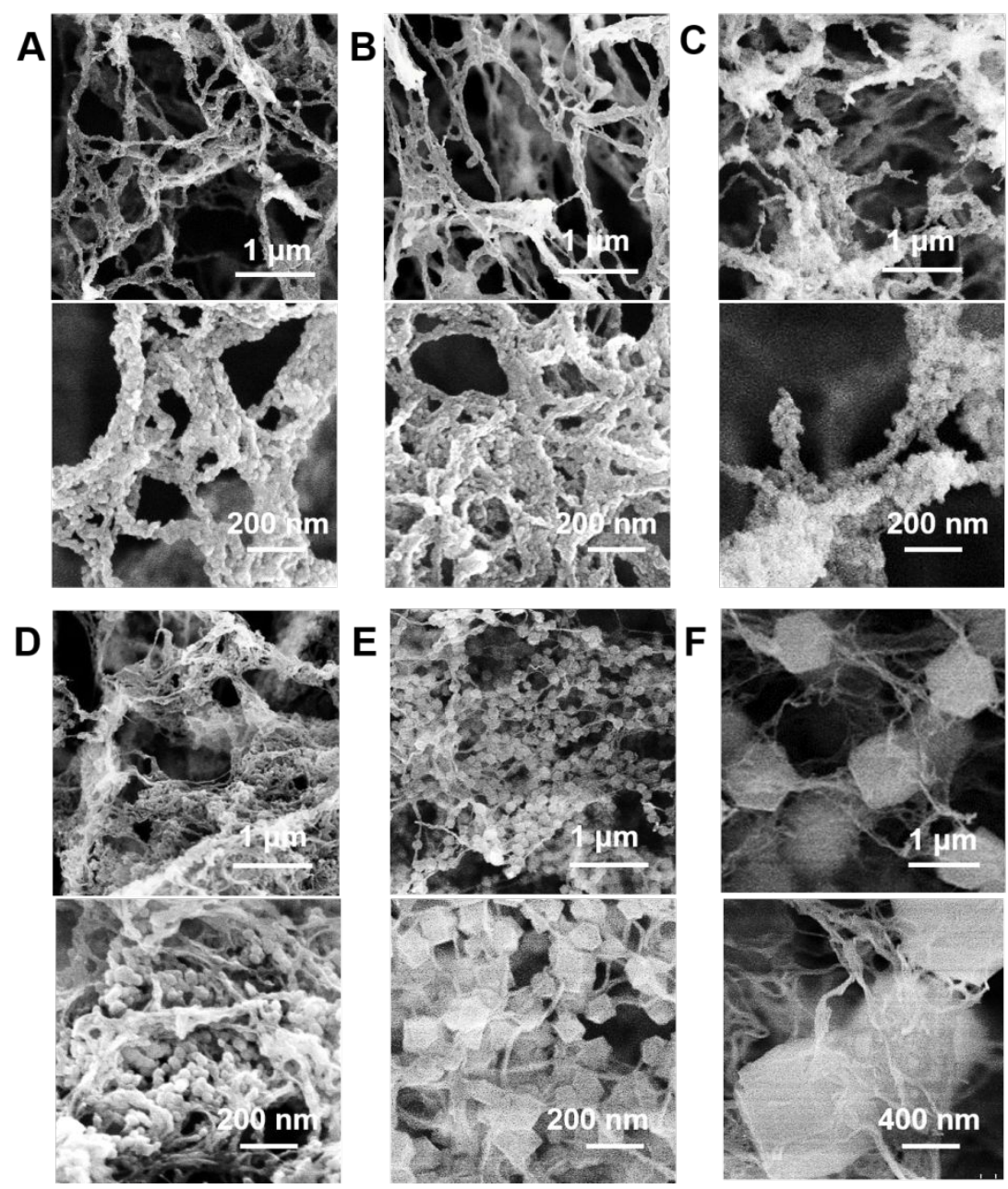

Figure S3. SEM images of ZIF-8 hybrid aerogels with different ZIF-8 contents: (A) 19 wt.\%, (B) 36 wt.\%, (C) 54 wt.\%, (D) 61 wt.\%, (E) 77 wt.\%, and (F) 84 wt.\%.
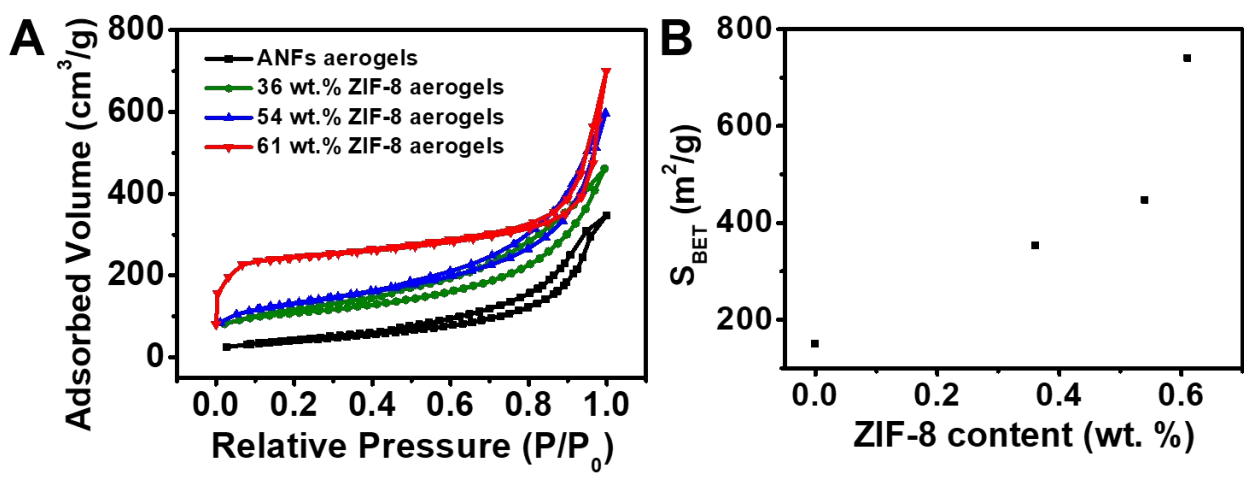

Figure S4. (A) Nitrogen adsorption-desorption analysis and (B) specific surface area of ZIF-8 hybrid aerogels. 


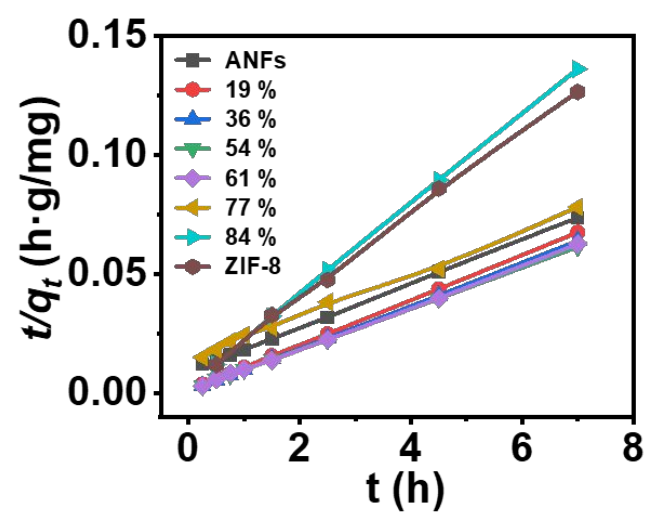

Figure S5. Pseudo second-order plots fitted with the data from Figure 3B (based on the mass of aerogels). $\mathrm{t} / \mathrm{q}_{\mathrm{t}}=1 /\left(\mathrm{kq}_{\mathrm{e}}{ }^{2}\right)+\mathrm{t} / \mathrm{q}_{\mathrm{e}}$, where $\mathrm{t}$ referred to the contact time $(\mathrm{h}), \mathrm{k}$ referred to the second-order rate constant $(\mathrm{g} /(\mathrm{mg} \cdot \mathrm{h})), \mathrm{q}_{\mathrm{e}}$ referred to the equilibrium uptake amount of dyes $(\mathrm{mg} / \mathrm{g}), \mathrm{q}_{\mathrm{t}}$ referred to the adsorption capacity at different times.
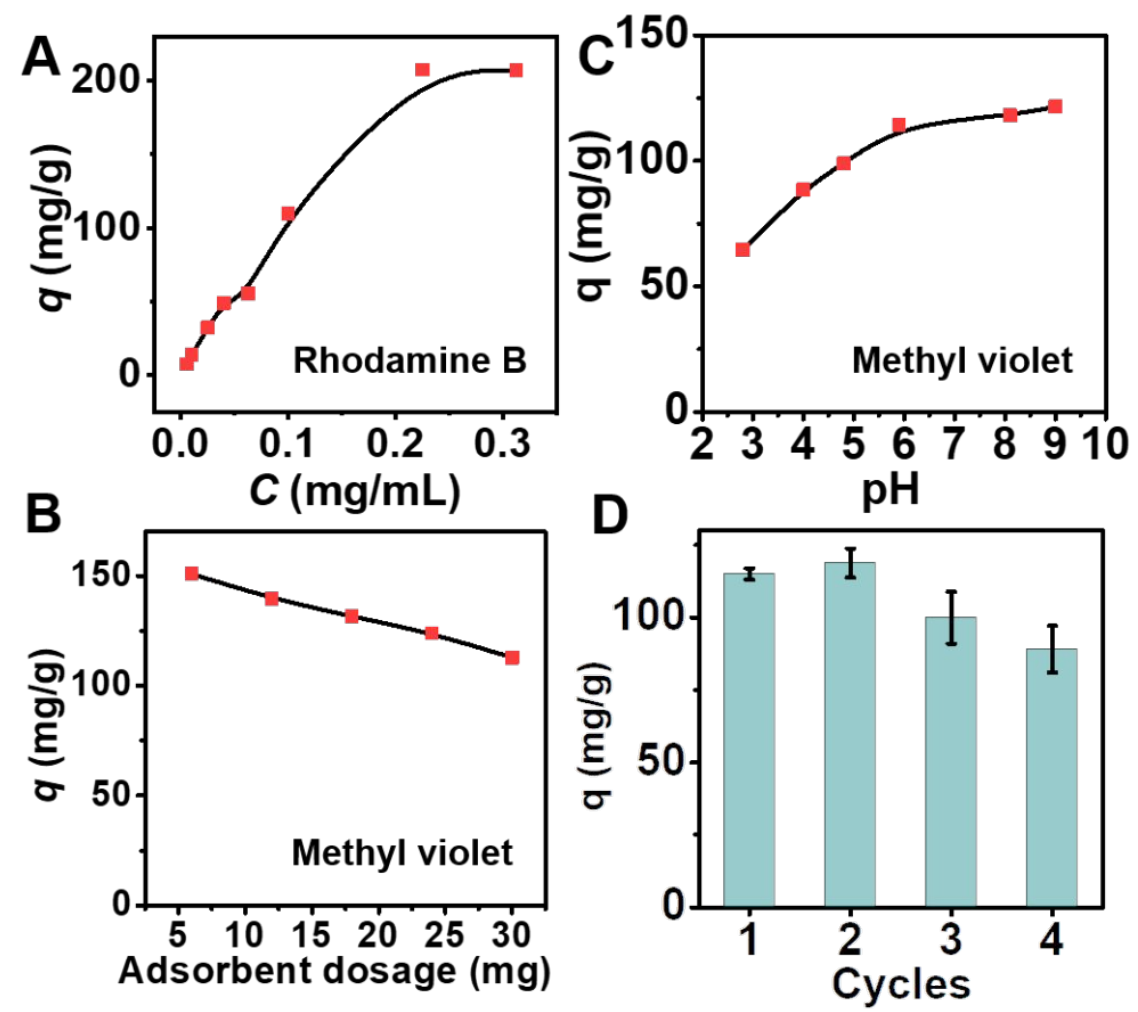

Figure S6. (A) Adsorption of rhodamine B versus its initial concentration, using ZIF-8 hybrid aerogels with 54 wt.\% ZIF-8 content. (B-C) Effect of (B) the amount of ZIF-8 aerogels and (C) $\mathrm{pH}$ on the adsorption of methyl violet. (D) The reusability of ZIF-8 aerogels. 


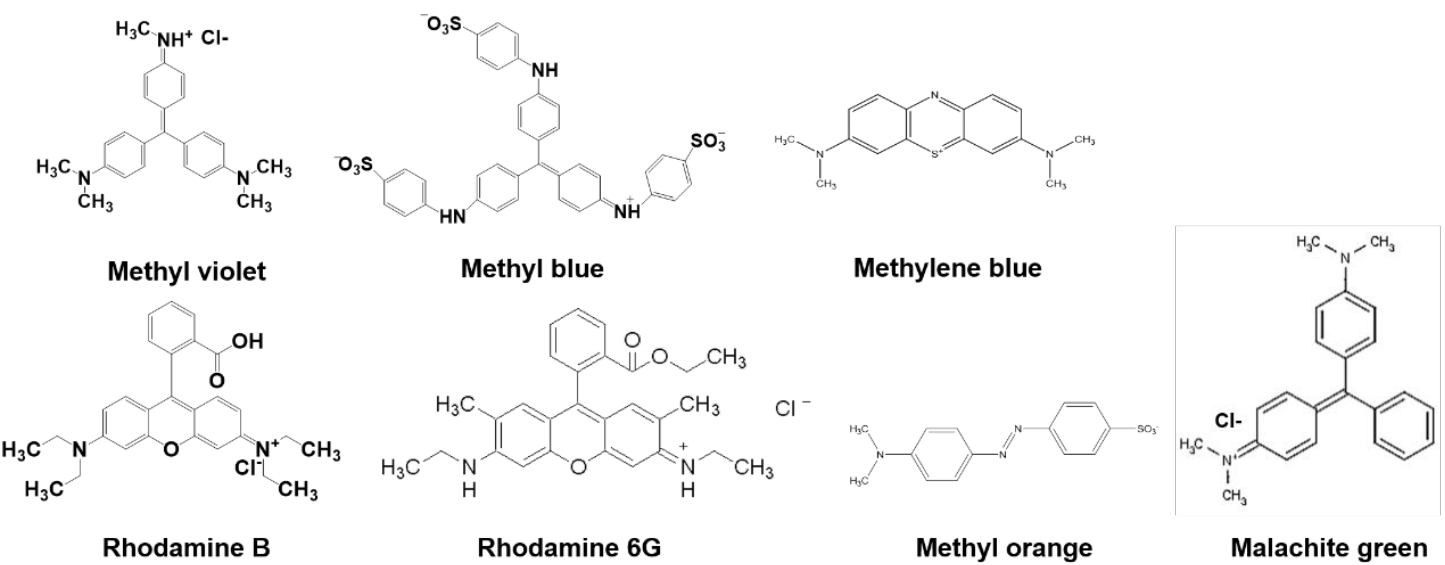

Figure S7. Molecular structures of dyes used.
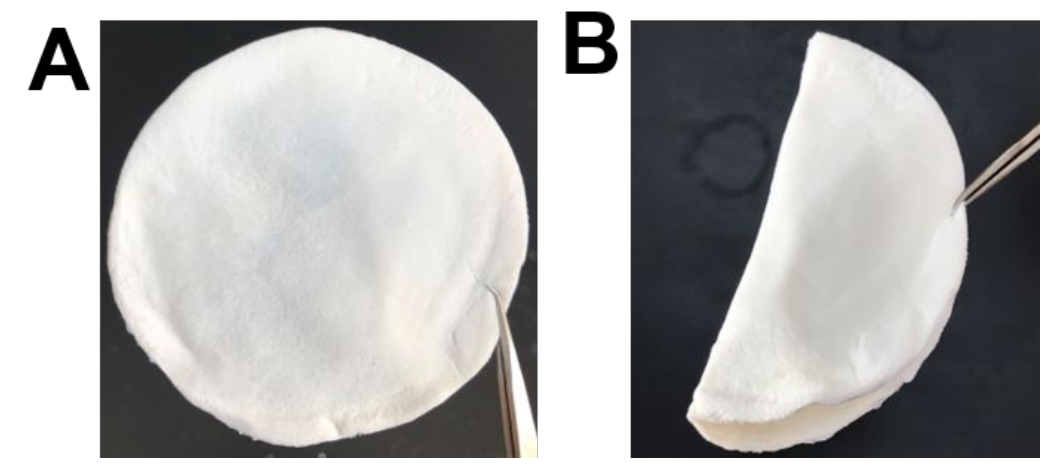

Figure S8. Photographs of (A) ZIF-8 (54 wt.\%) hybrid aerogel and (B) folded aerogel showing flexibility.
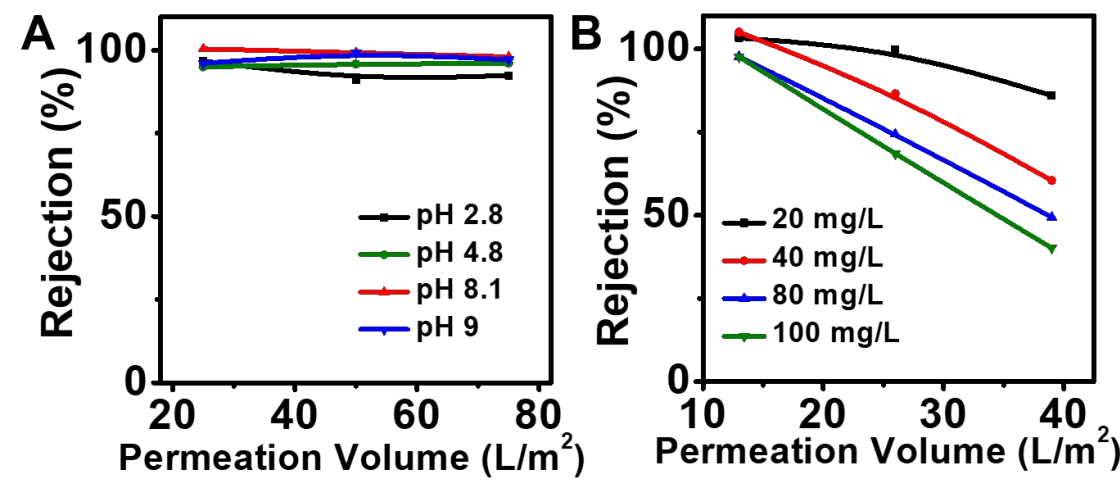

Figure S9. Effect of (A) $\mathrm{pH}$ and (B) the concentration of methyl violet on its rejection, under a pressure of 0.5 bar and aerogel thickness of $\sim 0.87 \mathrm{~mm}$. The concentration of methyl violet was $10 \mathrm{mg} / \mathrm{L}$ in (A). 


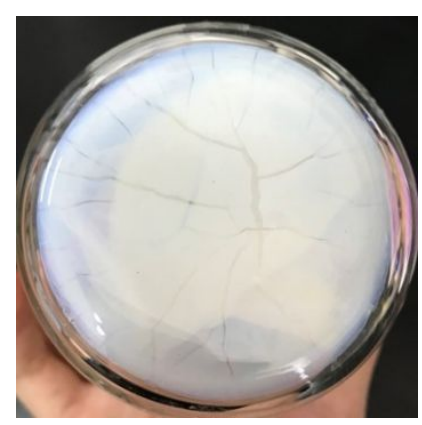

Figure S10. Photograph of ANFs aerogel with thickness of $\sim 0.87 \mathrm{~mm}$. It was fragile with lots cracks after the freeze-drying.
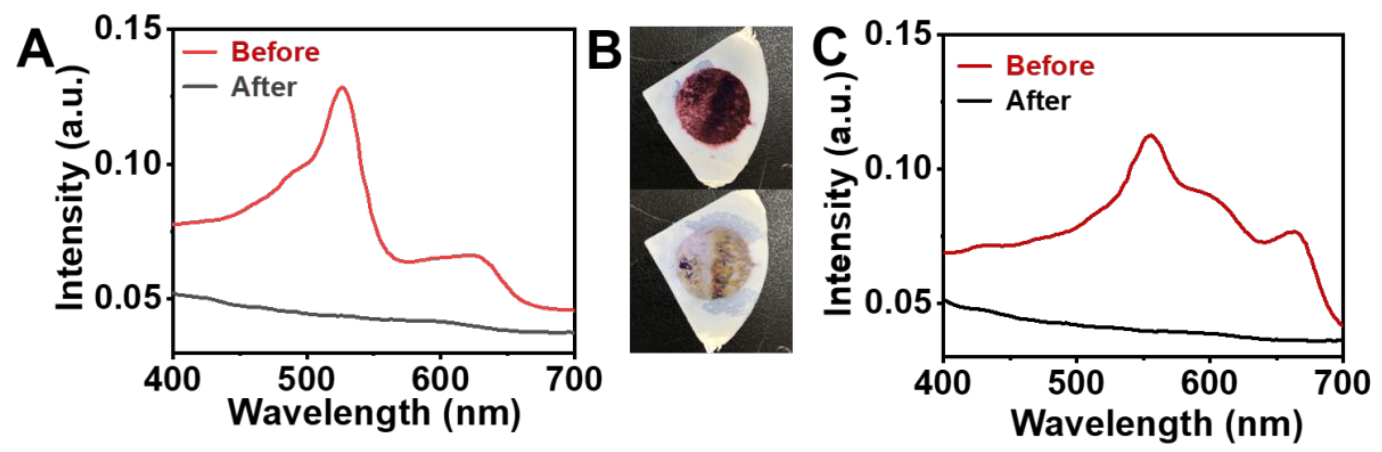

Figure S11. (A, C) Uv-vis spectra of mixed dyes before and after passing through the ZIF-8 (54 wt.\%) hybrid aerogels. (B) Photographs of the upper and nether sides of ZIF8 hybrid aerogels after filtration. Mixed dyes: (A) $1 \mathrm{mg} / \mathrm{L}$ methyl blue $+1 \mathrm{mg} / \mathrm{L}$ rhodamine $6 \mathrm{G}+1 \mathrm{mg} / \mathrm{L}$ methyl orange; (C) $1 \mathrm{mg} / \mathrm{L}$ methylene blue $+1 \mathrm{mg} / \mathrm{L}$ methyl violet $+1 \mathrm{mg} / \mathrm{L}$ rhodamine $\mathrm{B}+1 \mathrm{mg} / \mathrm{L}$ methyl orange $+1 \mathrm{mg} / \mathrm{L}$ malachite green . 
Table S1. Detailed synthesis conditions for ZIF-8 hybrid aerogels (Reactions were conducted in methanol at $25^{\circ} \mathrm{C}$ ).

\begin{tabular}{|c|c|c|c|}
\hline Sample ID & $\begin{array}{c}\mathrm{C}_{\mathrm{ANFs}} \text { gels } \\
(\mathrm{mg} / \mathrm{mL})\end{array}$ & $\begin{array}{c}\mathrm{C}_{\mathrm{Zn} 2+} \\
(\mathrm{mg} / \mathrm{mL})\end{array}$ & $\begin{array}{c}\mathrm{C}_{\text {ligand }} \\
(\mathrm{mg} / \mathrm{mL})\end{array}$ \\
\hline 19 wt. \% ZIF-8 aerogels & 0.8 & 0.2 & 0.44 \\
\hline 36 wt. \% ZIF-8 aerogels & 0.8 & 0.5 & 1.1 \\
\hline 54 wt. \% ZIF-8 aerogels & 0.8 & 5 & 11 \\
\hline 61 wt. \% ZIF-8 aerogels & 0.8 & 10 & 22 \\
\hline 77 wt. \% ZIF-8 aerogels & 0.8 & 30 & 65.7 \\
\hline 84 wt. \% ZIF-8 aerogels & 0.8 & 50 & 110 \\
\hline
\end{tabular}

Table S2. Adsorption capacity of ZIF-8 hybrid aerogels for methyl violet compared with other reported MOFs-based systems.

\begin{tabular}{|c|c|c|c|c|c|}
\hline Adsorbent & $\begin{array}{c}\text { Dye } \\
\text { concentration } \\
(\mathrm{mg} / \mathrm{L})\end{array}$ & Kinetics & $\begin{array}{l}\text { Second-order } \\
\text { rate constant } \\
\left(\mathrm{g} \cdot \mathrm{mg}^{-1} \cdot \mathrm{h}^{-1}\right)\end{array}$ & $\begin{array}{c}\text { Experimental } \\
\text { adsorption } \\
\text { capacity } \\
(\mathrm{mg} / \mathrm{g}) \\
\end{array}$ & $\begin{array}{c}\text { Refere } \\
\text { nces }\end{array}$ \\
\hline ZIF-8 hybrid aerogels & 100 & $\begin{array}{l}\text { pseudo- } \\
\text { second- } \\
\text { order }\end{array}$ & 0.55 & 113.7 & $\begin{array}{l}\text { This } \\
\text { work }\end{array}$ \\
\hline $\begin{array}{l}\text { ZIF-8 templated with } \\
\text { nanocellulose } \\
\text { aerogels }\end{array}$ & 10 & $\begin{array}{l}\text { pseudo- } \\
\text { second- } \\
\text { order }\end{array}$ & & $\sim 27$ & 1 \\
\hline $\begin{array}{l}\text { Multi-walled carbon } \\
\text { nanotubes } / \mathrm{Mn}_{0.8} \mathrm{Zn}_{0.2} \mathrm{~F} \\
\mathrm{e}_{2} \mathrm{O}_{4} \text { nanoparticles }\end{array}$ & 5 & $\begin{array}{l}\text { pseudo- } \\
\text { second- } \\
\text { order }\end{array}$ & 0.028 & 5 & 2 \\
\hline $\begin{array}{l}\text { Reduced graphene } \\
\text { oxide/ZIF-67 aerogel }\end{array}$ & 500 & $\begin{array}{l}\text { pseudo- } \\
\text { second- } \\
\text { order }\end{array}$ & & 1714.2 & 3 \\
\hline $\begin{array}{l}\text { Reduced graphene } \\
\text { oxide/ZIF-67 aerogel }\end{array}$ & 100 & $\begin{array}{l}\text { pseudo- } \\
\text { second- } \\
\text { order }\end{array}$ & & $\sim 200$ & 3 \\
\hline $\begin{array}{c}\text { Activated carbon- } \\
\text { HKUST-1-MOF } \\
\text { hybrid }\end{array}$ & 10 & $\begin{array}{l}\text { pseudo- } \\
\text { second- } \\
\text { order }\end{array}$ & 0.69 & 18.25 & 4 \\
\hline $\begin{array}{l}\text { Copper coordination } \\
\text { polymer with } \\
\text { dithiooxamide } \\
\left(\mathrm{H}_{2} \mathrm{dtoaCu}\right)\end{array}$ & 100 & $\begin{array}{l}\text { pseudo- } \\
\text { second- } \\
\text { order }\end{array}$ & 0.222 & 68.01 & 5 \\
\hline $\begin{array}{c}\text { Anionic zeolite-like } \\
\text { metal-organic } \\
\text { framework } \\
{\left[\left(\mathrm{CH}_{3}\right)_{2} \mathrm{NH}_{2}\right]_{6}\left[\mathrm{Sr}_{13}(\mathrm{O})\right.} \\
\end{array}$ & 1000 & & & 184 & 6 \\
\hline
\end{tabular}




\begin{tabular}{|c|c|c|c|c|c|}
\hline $\begin{array}{c}{ }_{3}(\mathrm{BTTC})_{8}(\mathrm{OH})_{2}\left(\mathrm{H}_{2} \mathrm{O}\right) \\
16] \cdot \mathrm{SS}\end{array}$ & & & & \\
\hline $\mathrm{UPC}-102-\mathrm{Zr}$ & $4 \times 10^{-5} \mathrm{~mol} / \mathrm{L}$ & & & 182 & 7 \\
\hline
\end{tabular}

Table S3. Flux and rejection of ZIF-8 hybrid aerogels during the filtration of dyes compared with other reported MOFs-based systems.

\begin{tabular}{|c|c|c|c|c|c|}
\hline Samples & Dyes & $\begin{array}{c}\text { Dye } \\
\text { concentration }\end{array}$ & $\begin{array}{c}\text { Flux } \\
\left(\mathrm{L} \cdot \mathrm{m}^{-2} \cdot \mathrm{h}^{-1} \cdot \mathrm{bar}^{-1}\right)\end{array}$ & Rejection & $\begin{array}{c}\text { Referenc } \\
\text { es }\end{array}$ \\
\hline $\begin{array}{l}\text { ZIF-8 hybrid } \\
\text { aerogels }\end{array}$ & methyl violet & $10 \mathrm{mg} / \mathrm{L}$ & 650 & $>97 \%$ & $\begin{array}{l}\text { This } \\
\text { work }\end{array}$ \\
\hline $\begin{array}{c}\text { Cellulose } \\
\text { nanofibrous } \\
\text { membrane with } \\
\text { ZIF-8 }\end{array}$ & janus green $B$ & $10 \mathrm{mg} / \mathrm{L}$ & $85.8 \mathrm{~L} \cdot \mathrm{m}^{-2} \mathrm{~h}^{-1}$ & $>97 \%$ & 8 \\
\hline $\begin{array}{l}\text { Partial reduction } \\
\text { graphene oxide } \\
\text { membrane }\end{array}$ & $\begin{array}{l}\text { methylene } \\
\text { blue }\end{array}$ & $\begin{array}{c}0.01-0.05 \\
\mathrm{mM}\end{array}$ & 98.5 & $99.3 \%$ & 9 \\
\hline $\begin{array}{c}\text { UiO-66-(COOH })_{2} / \\
\text { partial reduction } \\
\text { graphene oxide } \\
\text { membrane }\end{array}$ & rhodamine B & $\begin{array}{c}0.01-0.05 \\
\mathrm{mM}\end{array}$ & $93.0-95.0$ & & 9 \\
\hline $\begin{array}{l}\text { UiO- } 66 \text { on } \\
\text { alumina membrane }\end{array}$ & humic acid & $1000 \mathrm{mg} / \mathrm{L}$ & 34 & $99 \%$ & 10 \\
\hline $\begin{array}{l}\text { Hydrophilic } \\
\text { regenerated } \\
\text { cellulose support } \\
\text { membrane with } \\
\text { UiO-66 } \\
\text { nanoparticles } \\
\end{array}$ & $\begin{array}{l}\text { methylene } \\
\text { blue }\end{array}$ & $1.0 \mu \mathrm{mol}$ & 785.8 & $100 \%$ & 11 \\
\hline $\begin{array}{l}\mathrm{MOF} / \mathrm{GO} \\
\text { membrane }\end{array}$ & $\begin{array}{c}\text { methyl } \\
\text { orange, } \\
\text { direct red } 80\end{array}$ & $200 \mathrm{mg} / \mathrm{L}$ & $\sim 16 \mathrm{~kg} \cdot \mathrm{m}^{-2} \mathrm{~h}^{-1}$ & $\sim 97 \%$ & 12 \\
\hline $\begin{array}{c}\text { NH2-UiO-66 PE } \\
\text { MMM- } 86 \%\end{array}$ & orange $\mathrm{G}$ & $100 \mathrm{mg} / \mathrm{L}$ & 115.9 & $99 \%$ & 13 \\
\hline $\begin{array}{c}\text { NH2-UiO-66 PE } \\
\text { MMM- } 86 \%\end{array}$ & fuchsine acid & $100 \mathrm{mg} / \mathrm{L}$ & 111.4 & $99.1 \%$ & 13 \\
\hline
\end{tabular}

References:

(1) Zhu, L.; Zong, L.; Wu, X.; Li, M.; Wang, H.; You, J.; Li, C. Shapeable Fibrous Aerogels of Metal-Organic-Frameworks Templated with Nanocellulose for Rapid and Large-Capacity Adsorption. ACS Nano 2018, 12 (5), 4462-4468, DOI: 10.1021/acsnano.8b00566.

(2) Gabal, M. A.; Al-Harthy, E. A.; Al Angari, Y. M.; Salam, M. A. MWCNTs Decorated with $\mathrm{Mn}_{0.8} \mathrm{Zn}_{0.2} \mathrm{Fe}_{2} \mathrm{O}_{4}$ Nanoparticles for Removal of Crystal-Violet Dye from Aqueous Solutions. Chem Eng $J$ 2014, 255, 156-164, DOI: 10.1016/j.cej.2014.06.019.

(3) Yang, Q. X.; Lu, R.; Ren, S. S.; Chen, C. T.; Chen, Z. J.; Yang, X. Y. Three Dimensional Reduced Graphene Oxide/ZIF-67 Aerogel: Effective Removal Cationic and Anionic Dyes from Water. Chem Eng J 2018, 348, 202-211, DOI: 10.1016/j.cej.2018.04.176.

(4) Azad, F. N.; Ghaedi, M.; Dashtian, K.; Hajati, S.; Pezeshkpour, V. Ultrasonically Assisted Hydrothermal Synthesis of Activated Carbon-HKUST-1-MOF Hybrid for Efficient Simultaneous 
Ultrasound-Assisted Removal of Ternary Organic Dyes and Antibacterial Investigation: Taguchi Optimization. Ultrason Sonochem 2016, 31, 383-393, DOI: 10.1016/j.ultsonch.2016.01.024.

(5) Li, X.; Zheng, L.; Huang, L.; Zheng, O.; Lin, Z.; Guo, L.; Qiu, B.; Chen, G. Adsorption Removal of Crystal Violet from Aqueous Solution Using a Metal-Organic Frameworks Material, Copper Coordination Polymer with Dithiooxamide. J Appl Polym Sci 2013, 129 (5), 2857-2864, DOI: 10.1002/app.39009.

(6) Shen, Y.; Fan, C.-C.; Wei, Y.-Z.; Du, J.; Zhu, H.-B.; Zhao, Y. An Anionic Zeolite-Like MetalOrganic Framework (AZMOF) with Moravia Network for Organic Dyes Absorption through Cation-Exchange. Dalton Trans 2016, 45, 10909-10915, 10.1039.C6DT01697A.

(7) Fan, W.; Wang, X.; Xu, B.; Wang, Y.; Liu, D.; Zhang, M.; Shang, Y.; Dai, F.; Zhang, L.; Sun, D. Amino-Functionalized MOFs with High Physicochemical Stability for Efficient Gas Storage/Separation, Dye Adsorption and Catalytic Performance. J Mater Chem A 2018, 6 (47), 24486-24495, DOI: 10.1039/c8ta07839d.

(8) Song, Y.; Seo, J. Y.; Kim, H.; Beak, K.-Y. Structural control of Cellulose Nanofibrous Composite Membrane with Metal Organic Framework (ZIF-8) for Highly Selective Removal of Cationic Dye. Carbohyd. Polym. 2019, 222, 115018-115018, DOI: https://doi.org/10.1016/j.carbpol.2019.115018.

(9) Zhang, P.; Gong, J.-L.; Zeng, G.-M.; Song, B.; Liu, H.-Y.; Huan, S.-Y.; Li, J. Ultrathin Reduced Graphene Oxide/MOF Nanofiltration Membrane with Improved Purification Performance at Low Pressure. Chemosphere 2018, 204, 378-389, DOI: https://doi.org/10.1016/j.chemosphere.2018.04.064.

(10) Abdullah, N.; Rahman, M. A.; Dzarfan Othman, M. H.; Jaafar, J.; Aziz, A. A. Preparation, Characterizations and Performance Evaluations of Alumina Hollow Fiber Membrane Incorporated with UiO-66 Particles for Humic Acid Removal. J. Membrane Sci. 2018, 563, 162-174, DOI: https://doi.org/10.1016/j.memsci.2018.05.059.

(11) Trinh, D. X.; Tran, T. P. N.; Taniike, T. Fabrication of New Composite Membrane Filled with UiO-66 Nanoparticles and Its Application to Nanofiltration. Sep. Purif. Technol. 2017, 177, 249256, DOI: https://doi.org/10.1016/j.seppur.2017.01.004.

(12) Ma, J.; Guo, X.; Ying, Y.; Liu, D.; Zhong, C. Composite Ultrafiltration Membrane Tailored by MOF@GO with Highly Improved Water Purification Performance. Chem. Eng. J. 2017, 313, 890898, DOI: https://doi.org/10.1016/j.cej.2016.10.127.

(13) Wang, H.; Zhao, S.; Liu, Y.; Yao, R.; Wang, X.; Cao, Y.; Ma, D.; Zou, M.; Cao, A.; Feng, X.; Wang, B. Membrane Adsorbers with Ultrahigh Metal-Organic Framework Loading for High Flux Separations. Nat. Commun. 2019, 10 (1), 4204-4204, DOI: 10.1038/s41467-019-12114-8. 